\title{
Design and Modeling of a Flexible Conductive Fabric Antenna Integrated in an OLED Light Source for WIMAX Wireless Communication Systems
}

\author{
Soukaina Sekkal ${ }^{1 *}$, Laurent Canale ${ }^{2 *}$, Mustapha El Halaoui ${ }^{1,2}$, Othmane Bendaou ${ }^{1}$, Adel Asselman ${ }^{1}$ \\ ${ }^{1}$ Optics and Photonics Laboratory, Université Abdelmalek Essaadi, Tetouan, Morocco \\ ${ }^{2}$ LAPLACE, Université de Toulouse, CNRS, INPT, UPS, 118 Route de Narbonne, 31062 Toulouse, France \\ Email: *sekkalsoukaina92@gmail.com, laurent.canale@laplace.univ-tlse.fr
}

How to cite this paper: Sekkal, S., Canale, L., El Halaoui, M., Bendaou, O. and Asselman, A. (2021) Design and Modeling of a Flexible Conductive Fabric Antenna Integrated in an OLED Light Source for WIMAX Wireless Communication Systems. Optics and Photonics Journal, 11, 413-429.

https://doi.org/10.4236/opj.2021.119030

Received: August 15, 2021

Accepted: September 23, 2021

Published: September 26, 2021

Copyright $\odot 2021$ by author(s) and Scientific Research Publishing Inc. This work is licensed under the Creative Commons Attribution International License (CC BY 4.0).

http://creativecommons.org/licenses/by/4.0/

\begin{abstract}
This work presents a new bendable antenna for worldwide interoperability for microwave access (WiMAX) wireless communication systems. These antennas, transparent and flexible, will be easily integrated into various media and in particular OLED lighting which could be part of the public lighting network of tomorrow as well as on all display media. The integration of these antennas as close as possible to the end-user is a possible solution to reduce the energy consumption which goes hand in hand with the increase in the data rate. This kind of new antenna, designed to be integrated in organic lightemitting diode (OLED), was modeled from a transparent VeilShieldTM conductive fabric and was placed on a $100 \%$ polyester substrate with a thickness of $1.5 \mathrm{~mm}$ and a loss tangent of 0.02 . We have tested and evaluated the characteristic parameters of our antenna, namely the reflection coefficient, the radiation pattern and the gain, to find out the performance of our proposed design. The performance of the transparent conductive fabric integrated in the $100 \%$ polyester substrate is tested for the application of flexible antenna operating at $3.5 \mathrm{GHz}$ with a gain value of $5.38 \mathrm{~dB}$. We have integrated this proposed new antenna with the OLED light source containing four layers of different materials and electrical properties: aluminum cathode layer, polymer layer, indium tin oxide (ITO) anode layer and glass substrate layer. After integration, the resonant frequency shifted to $3.52 \mathrm{GHz}$ with a gain value of 4.61 $\mathrm{dB}$. In addition, we also tested the concave bending on the reflection coefficient of the proposed flexible antenna taking into account the different bending angles. This work demonstrates the possibility of integrating these unconventional materials used for the proposed antenna within the OLED despite
\end{abstract}


weak effects on the resonant frequency and the gain of the proposed antenna after integration.

\section{Keywords}

Flexible Antenna, Organic Light-Emitting Diode, Transparent Conductive Fabric, Wireless Communication Systems

\section{Introduction}

For several decades and in particular with the appearance of cell phones, integrated antennas have given rise to significant research work. With increasingly important data rates which will lead to a multiplication and diversification of communication channels, this research in the field of microwaves and antenna technology is becoming more and more demanding. At the same time, research in the field of organic light-emitting devices (OLED) has also accelerated recently. OLED technology is used for display in the field of flat screens and as well as their use as lighting panel where the electroluminescent properties of OLEDs do not require backlighting [1]. In addition, this technology has excellent contrast and excellent brightness with strong integration (thin screen) and high energy efficiency.

The flexibility of these materials also offers the possibility of producing extremely thin flexible screens, potentially curved, flexible or even rollable and therefore capable of integrating them on a wide variety of supports such as plastics or fabrics. Forecast papers predict the development of smart windows where these latest will integrate OLED, as well as other versatile functions such as solar power generation [2] [3]. This OLED technology will certainly be the next generation of light sources because with wide and diffused lighting, it does not induce glare unlike LEDs, with in addition a high quality of light, instant ignition, low energy consumption and simple and inexpensive manufacturing processes [4].

Recently, work has been published which has mainly focused on the integration of optically transparent antennas into the new technology of the OLED light source either for the applications for Wi-Fi and Bluetooth connectivity [5] [6] or for 5G applications [7] [8]. All these studies and works show the applicability of optically transparent antennas in OLED light sources. With the same idea of integration, we show for the first time the design and modeling of an antenna made from a flexible conductive fabric integrated into an OLED light source.

To our knowledge, this is the first time that flexible antennas based on transparent conductive fabric have been integrated into OLED light sources, so this article can serve as a reference. The idea is to use the wide distribution of domestic and urban lighting within cities to ensure a high flow rate by limiting the powers emitted by the transmitter and the receiver because the distance between the user and the source remains short. In addition, with these advantages (reduction in energy consumption by enabling broadband), these antennas can also be in- 
tegrated on flexible and transparent supports.

In this study, we simulated the radiation of this transparent conductive fabric VeilShield ${ }^{\mathrm{TM}}$ antenna and determined its different characteristics. This patch antenna has been developed to operate at high gain of $5.38 \mathrm{~dB}$ and at a frequency of $3.5 \mathrm{GHz}$ for use in WiMAX wireless communication systems. This paper is based on the improvement of our previously published work [9]. This work is structured as follows: after the introduction, the second part shows a description of the proposed antenna and its materials used in this design are presented; in part III, the simulation study of the flexible fabric made antenna is presented and the results of the simulation are discussed (from parametric study, radiation diagram, reflection coefficient and gain), which gave results suited to the initially targeted objective and which guarantees the possibility of designing a new flexible antenna with unconventional materials. In the last part before the conclusion, this flexible antenna is integrated into the OLED light source structure which is composed of four simple layers by analyzing their performance after this integration by the simulation of their parameters by focusing the study on the reflection coefficient, the radiation diagram and the concave bending for different angle values.

\section{Antenna Design and Material Characteristics}

Regarding the materials used for flexible antennas, there are very different technologies such as conductive wires or fabrics [10] [11], silver paste [12] or conductive inks [13] which make it possible to achieve the radiating element of the antenna. To constitute the flexible substrate, one can find polyester [14] or polymers using fibers such as cotton or felt [12] but also polydimethylsiloxane (PDMS) or graphene (FGF) [15] films.

These different materials have different costs and performance and must combine reliability, robustness, sturdiness and flexibility.

\subsection{Antenna Design}

This part presents the geometry and the different dimensions that are used to design the flexible antenna. It also presents the calculations which made it possible to determine these parameters.

This antenna, which also allows light to pass through, is made entirely of fabric and has been designed to operate at $3.5 \mathrm{GHz}$ with a reflection coefficient measured at $(|S 11|<-10 \mathrm{~dB})$, in order to demonstrate the concept of use of this type of fabric to design flexible antennas.

This antenna is designed on a $1.5 \mathrm{~mm}$ thick $100 \%$ polyester substrate and a loss tangent of 0.02 . The proposed antenna has a size of $55 \mathrm{~mm} \times 46 \mathrm{~mm}$ (LS $\times$ WS).

The dimensions of the microstrip feed line are calculated to ensure a $50 \Omega$ input impedance with length and width value $\left(L_{f}=14 \mathrm{~mm}, W_{f}=4.12 \mathrm{~mm}\right)$ respectively. In the antenna geometry, the ground plane covers the full rear part of 
the substrate to reduce back radiation thus to mitigate the harmful effects that are usually caused in communication applications on the body [16].

The width and length of the radiating patch are calculated using the equations given in [17].

The width of the microstrip patch antenna is given by:

$$
W_{p}=\frac{c}{2 * f_{r}} \sqrt{\frac{2}{\varepsilon_{r}+1}}
$$

The length of the patch is obtained by the following expression:

$$
L_{p}=\frac{c}{2 * f_{r} \sqrt{\varepsilon_{\text {eff }}}}-2 \Delta L
$$

where $\varepsilon_{\text {eff }}$ is the effective dielectric constant and $\Delta L$ is the length extension of the patch antenna are given by following Equations (3) and (4) respectively:

$$
\begin{gathered}
\varepsilon_{\text {eff }}=\frac{\varepsilon_{r}+1}{2}+\frac{\varepsilon_{r}-1}{2} \sqrt{\frac{1}{1+12 \frac{h}{W_{p}}}} \\
\Delta L=0.412 h \frac{\left(\varepsilon_{\text {eff }}+0.3\right)\left(\frac{W_{p}}{h}+0.264\right)}{\left(\varepsilon_{\text {eff }}-0.258\right)\left(\frac{W_{p}}{h}+0.8\right)}
\end{gathered}
$$

In order to design our patch antenna to operate at $3.5 \mathrm{GHz}$, a parametric study was carried out during geometric adjustment on different parameters such as the length and width of the patch and the substrate as well as the length of the slot of the proposed antenna. The results are used to optimize its parameters in order to obtain a better understanding of the behavior of the antenna at the frequency $3.5 \mathrm{GHz}$. Its structure and optimized parameters are presented in Figure 1 and Table 1 , respectively.

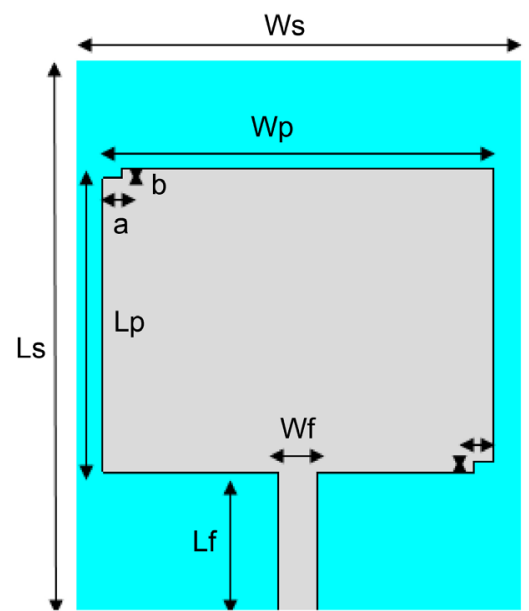

(a)

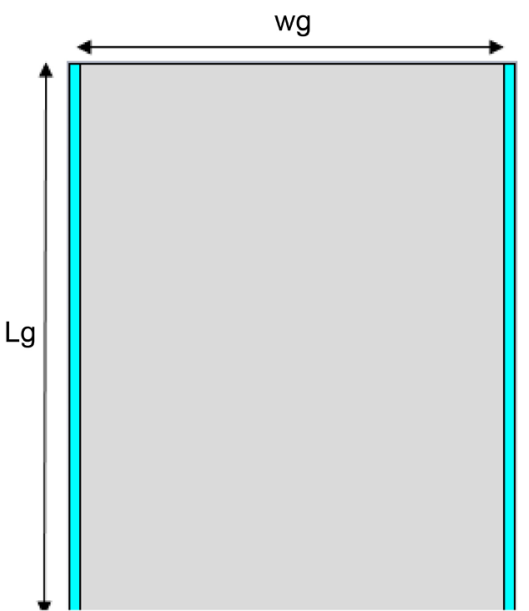

(b)

Figure 1. Geometry of the flexible antenna: (a) radiating patch; (b) ground plane. 
Table 1. Dimensions of the antenna.

\begin{tabular}{ccc}
\hline Parameter & Description & Value (mm) \\
\hline$W_{p}$ & width of the patch & 40 \\
$L_{p}$ & Length of the patch & 30 \\
$W_{g}$ & width of the ground & 40 \\
$L_{g}$ & Length of the ground & 55 \\
$a$ & Length of the slot & 2 \\
$b$ & width of the slot & 1 \\
\hline
\end{tabular}

\subsection{Material Characteristics}

Concerning the data used for the calculations relating to the radiating element and to the ground plane of the antenna, we used the electrical characteristics of the transparent conductive fabric (VeilShield ${ }^{\mathrm{TM}}$ ) provided by the manufacturer [18]. This conductive fabric is meshed from woven mesh polyester fibers coated with a blackened nickel/copper/zinc alloy to provide greater corrosion resistance while maintaining very good transparency. The surface resistance is very low and of the order of $0.089 \Omega / \mathrm{sq}$ with an electrical conductivity of $2.10^{5} \mathrm{~S} / \mathrm{m}$ and a thickness of $0.057 \mathrm{~mm}$. By using the Relation (1), we calculated the theoretical transparency of the fabric and its value is $73 \%$ which is close to the value from the datasheet of the manufacturer (70\%) [18].

$$
T \%=(p-s)^{2} / p^{2}
$$

where $T$ was theoretical transparency of the fabric in percentage, the $s$ was the strip width had a size of $0.09 \mathrm{~mm}$ and $p$ is the pitch of the mesh with a size of $0.62 \mathrm{~mm}$, as shown in Figure 2.

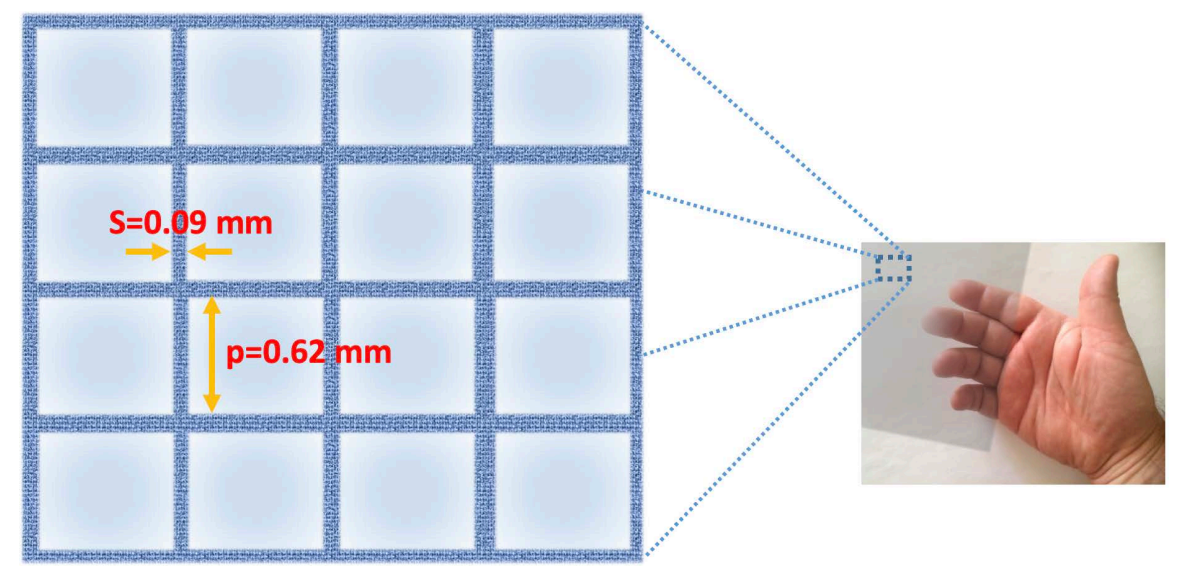

Figure 2. Transparent conductive fabric mesh dimensions [18].

In urban areas, microplastic fibers form a large part of microplastics. Synthetic textiles consider themselves to be a major source of microplastic fibers, in particular polyester, which is experiencing an increase in their use due to their good 
quantitative and qualitative reasons [19]. It is for these reasons that we have used an insulating element made of $100 \%$ polyester for our antenna. This fabric is in fact entirely composed of polyester threads or fibers with a dielectric constant of 2.85. In addition, this material has many advantages: it is flexible, very stable to humidity, high mechanical strength and elasticity to deformation of $40 \%$ [20].

\section{Results and Discussions}

The simulations and results presented in this part were carried out using the Ansys High Frequency Structure Simulator (Ansys-HFSS) software (version 1.4).

They relate to the reflection coefficient, the gain and the radiation pattern and they also present a parametric study of the performances of this structure as a function of the width of the slot "b".

\subsection{Parametric Study as Function of the Width of the Slot "b"}

In this part, we sought to analyze the influence of the dimensions of the antenna and in particular of the width of the slot " $b$ " on the reflection coefficient S11. In this parametric study, the other values given in Table 1 were held constant. The evolution of the reflection coefficient S11 with the variation of the width of the slot $b$ (for $b=1 \mathrm{~mm}, 3 \mathrm{~mm}$, and $5 \mathrm{~mm}$ ) is presented in Figure 3. The results show that this parameter does not have a strong influence on the frequency resonance of our antenna. Nevertheless, we can see that as the value of the width " $b$ " increases, the resonant frequency also increases and the value of the reflection coefficient S11 is less suitable (decreasing slightly).

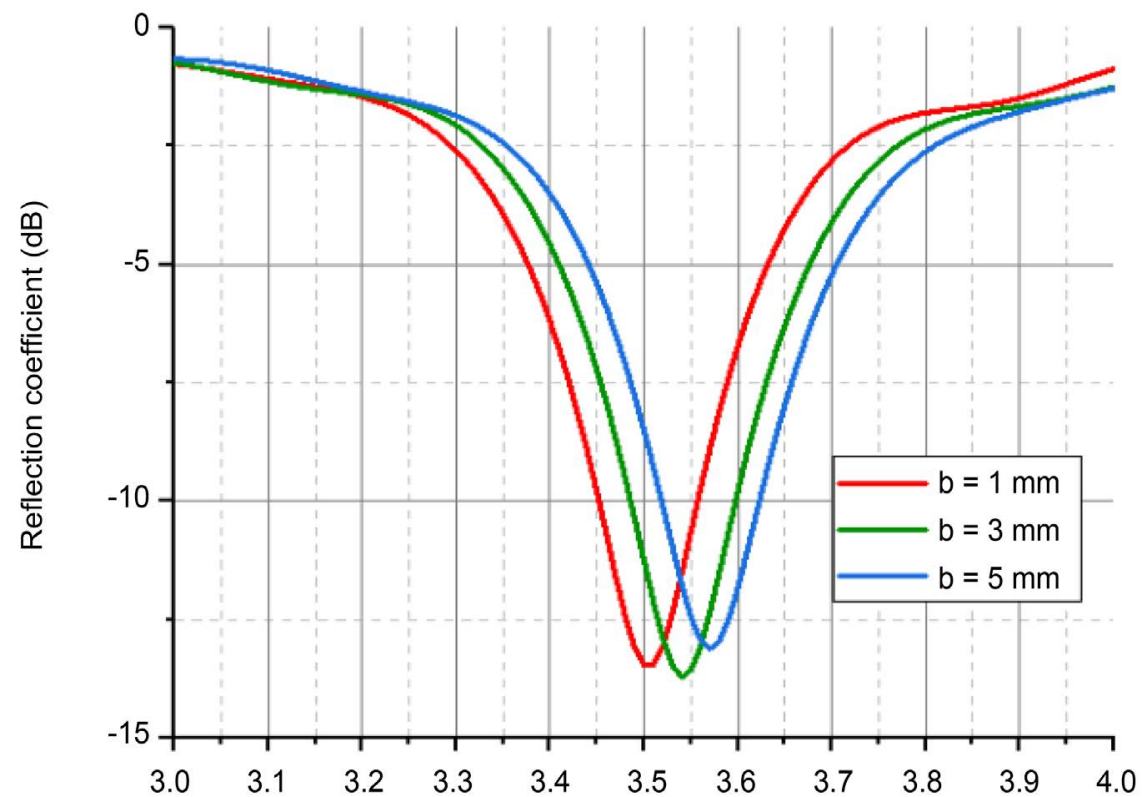

Figure 3. Reflection coefficient (S11) vs the width of the slot "b".

\subsection{Performances of the Antenna, Reflection Coefficient (S11)}

Figure 4 shows the performance of the antenna as a function of the reflection 
coefficient S11 with the dimensions given in Table 1.

The results obtained show over the entire operating frequency, at a resonance frequency of $3.5 \mathrm{GHz}$, a value of $\mathrm{S} 11$ less than $-10 \mathrm{~dB}$ which is appropriate for WIMAX wireless communication systems. In addition, the operating band is narrow and between $3.41 \mathrm{GHz}$ to $3.59 \mathrm{GHz}$ with a bandwidth of $0.18 \mathrm{GHz}$ which only applies to WIMAX wireless applications.

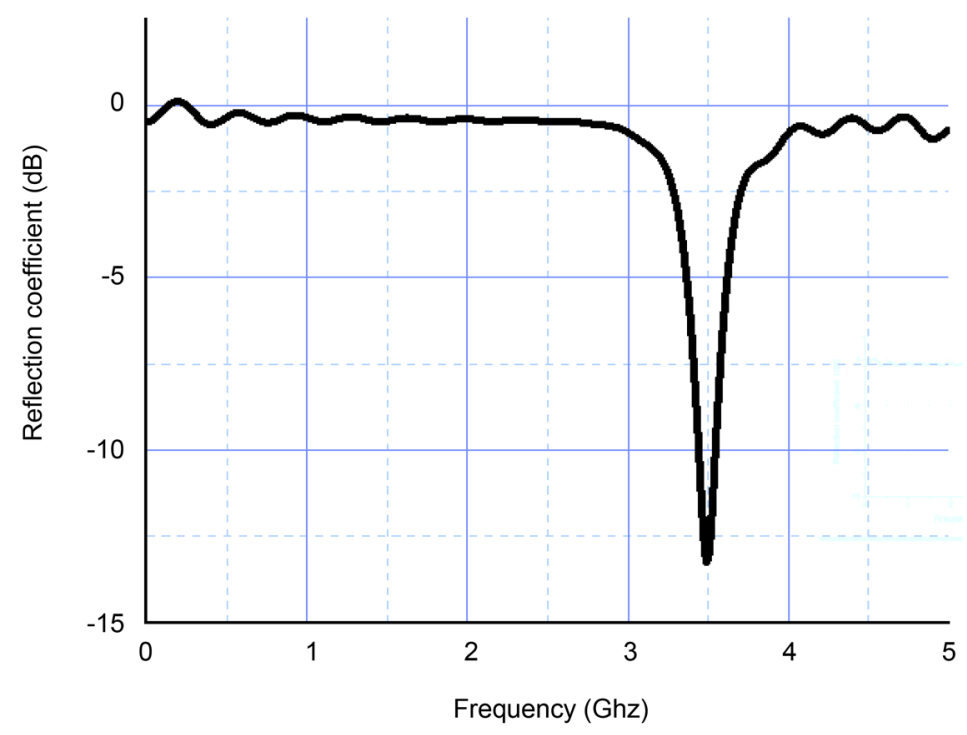

Figure 4. Performance of the flexible antenna (S11 reflection coefficient).

\subsection{Performances of the Antenna in Terms of Gain}

In order to quantify the amount of energy emitted by the flexible antenna, we simulated the gain. Figure 5 gives the results for a frequency band between 3 $\mathrm{GHz}$ and $3.8 \mathrm{GHz}$.

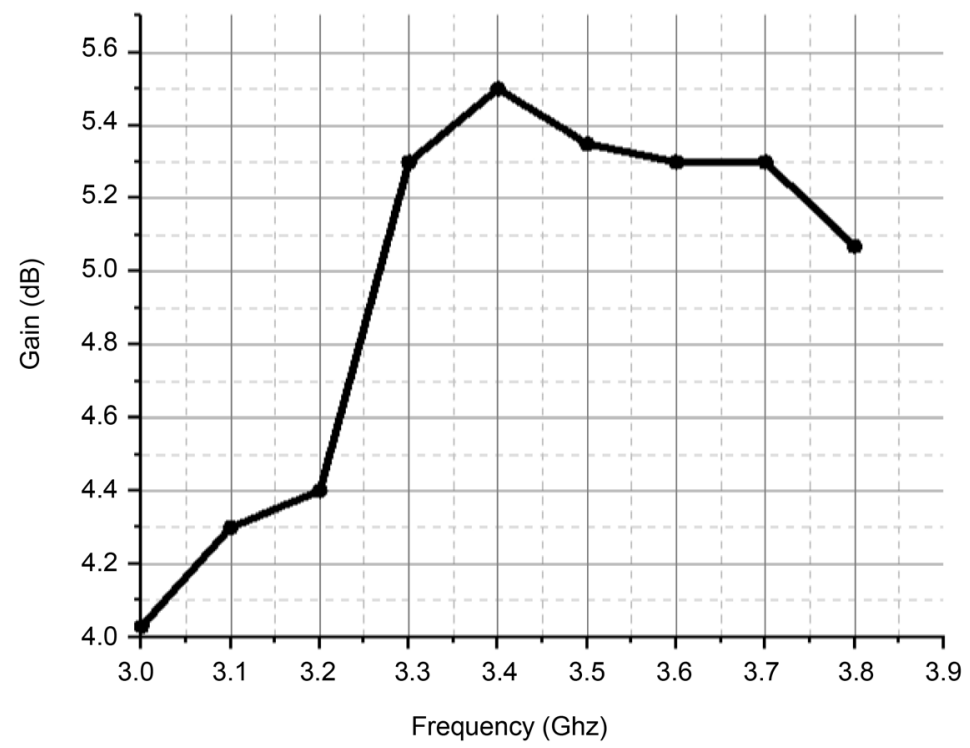

Figure 5. Performance of the proposed flexible antenna in terms of gain. 
We find that the maximum value obtained of $5.5 \mathrm{~dB}$ with a resonant frequency of $3.4 \mathrm{GHz}$ which does not correspond to our working frequency. However, our antenna has a high gain of $5.38 \mathrm{~dB}$ at the operating frequency of $3.5 \mathrm{GHz}$ with a gain very slightly lower than the value of the maximum gain.

The gain increases rapidly with the frequency between 3 and $3.4 \mathrm{GHz}$ but beyond this frequency, it drops slightly to stabilize around 3.6/3.7 $\mathrm{GHz}$ before dropping after $3.7 \mathrm{GHz}$.

Although the gain is higher at $3.4 \mathrm{GHz}$, overall, it is still very high on the 3.3 $\mathrm{GHz}-3.7 \mathrm{GHz}$ band with a gain of $5.38 \mathrm{~dB}$ at $3.5 \mathrm{GHz}$. Besides, by using an antenna array, it can be an effective solution to improve the gain of our flexible antenna.

\subsection{Presentation of the Simulation of 3D Radiation Pattern}

In order to assess the performance of the antenna, we simulated the far-field radiation pattern at $3.5 \mathrm{GHz}$. The results are shown in Figure 6.

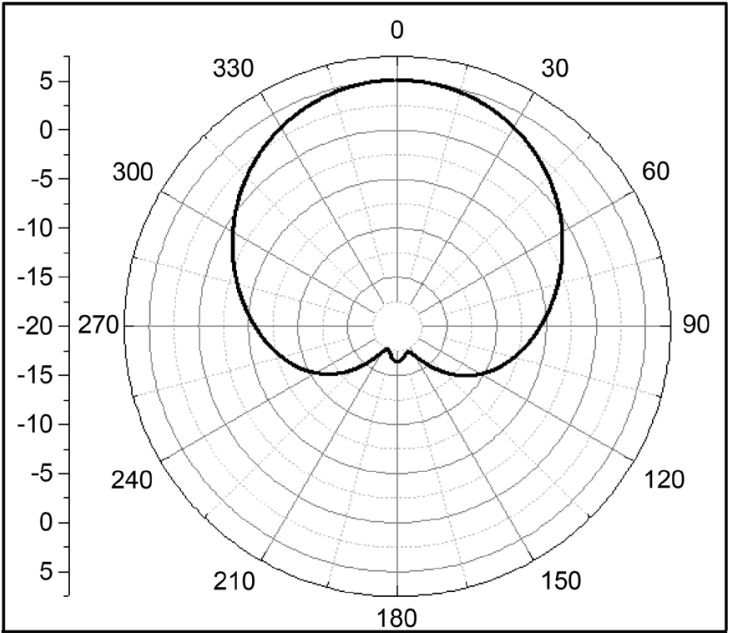

(a)

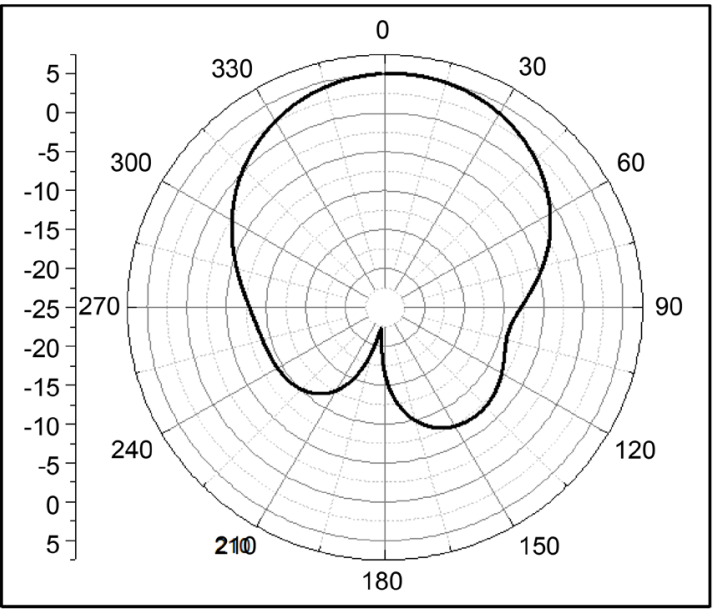

(b)

Figure 6. Radiation pattern at $3.5 \mathrm{GHz}$ of the proposed antenna for (a) E-plane and (b) H-plane. 
From this figure, the results obtained have demonstrated most of radiation is reflected back from the forward interface of the flexible proposed antenna and more specifically the strong far field radiation exists at the center of the radiating element with a maximum gain of $5.38 \mathrm{~dB}$ at $3.5 \mathrm{GHz}$ a stable power distribution at this resonant frequency which is suitable for WiMAX Wireless communication systems.

\section{Flexible Antenna Integration into an Organic Light Emitting Diode (OLED)}

OLEDs are monolithic semiconductor devices that commonly consist of thin organic films sandwiched between two thin film conductive electrodes (anode and cathode). As well as, the transparent conductive electrodes (TCE) are important composites of active display panels such as this technology organic light emitting diodes (OLED) [21] and liquid crystal displays (LCD).

The recent development of this technology concerning the design, manufacture and synthesis of materials at the nanoscale has included new transparent conductive electrodes (TCE) ranging from graphene [22], conductive polymers [23] to nanowires silver [24]. Among these transparent conductive materials, we find transparent and conductive oxides (TCO) which exhibit high electrical conductivity and good transparency in the visible range. The most widely used of these materials is Tin Doped Indium Oxide (ITO) because it has moderate sheet resistance and it offers great brittleness to the film, which eventually makes it suitable for future flexible and transparent portable electronic devices.

\subsection{Geometry of the Flexile Antenna Integrated in OLED Light Source Structure}

After having simulated the flexible antenna alone, this part presents the integration of the studied antenna in an OLED light source. Figure 7 shows a simplified

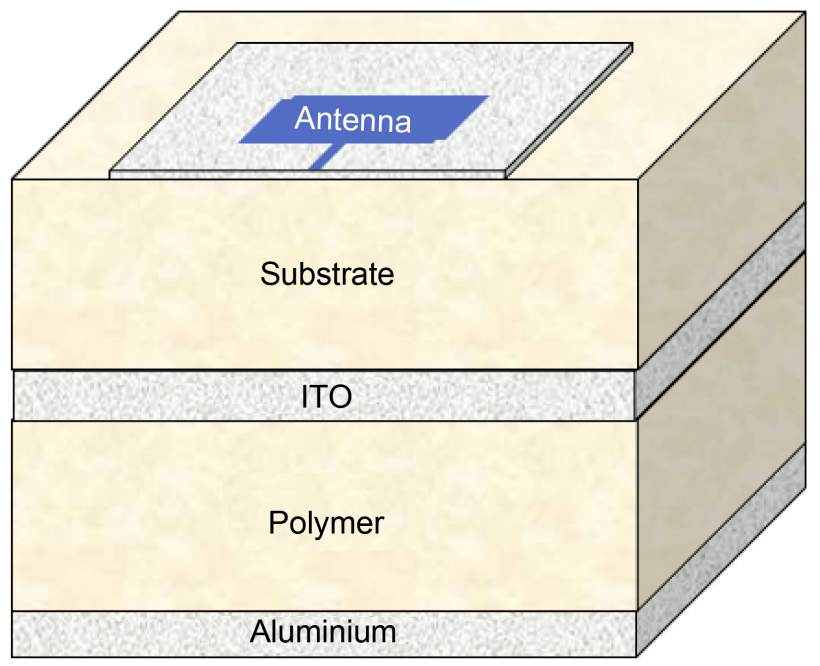

Figure 7. Simplified OLED light source structure with flexible antenna. 
version of a sectional view of an organic LED. The "polymer" layer is made up of several layers (Hole transport layer, Electron transport layer, emissive layer, etc.) which are not shown here. In general, an OLED consists of an organic layer between two electrodes (Aluminum and Indium Tin Oxide (ITO), the whole being encapsulated in one by a more or less flexible structure (glass or polymer Substrate). The antenna is located on top of the OLED (technical characteristics given in [25]) with the dimensions of $130.2 \times 47.8 \mathrm{~mm}^{2}$. The different layers have the following thicknesses: the aluminum cathode, $120 \mathrm{~nm}$, the polymer, $270 \mathrm{~nm}$, the ITO electrode, $150 \mathrm{~nm}$ and the substrate of the order of $215 \mathrm{~nm}$.

The principle of study in this section is to integrate the flexible antenna to OLED light sources without affecting their performance and by analyzing their characteristics among which the reflection coefficient, the gain after this integration and bending.

\subsection{Evaluation of the Reflection Coefficient (S11) of the Antenna including the OLED Structure}

Figure 8 superimposes the results obtained for the reflection coefficient (S11) without, and then with the OLED. These results show that the presence of the OLED affects the performance of the antenna with a shift of the resonant frequency to a higher frequency.

The new resonance frequency is $3.52 \mathrm{GHz}$ with the same bandwidth found before integration. These results show that the input impedance of the antenna is no longer suited to $50 \Omega$.

These changes are due to the influence of organic materials in the OLED on the antenna. However, this variation in the resonance frequency does not induce a strong modification and the value of the coefficient S11 remains acceptable, which makes it possible to validate the integration of the antenna in the device.

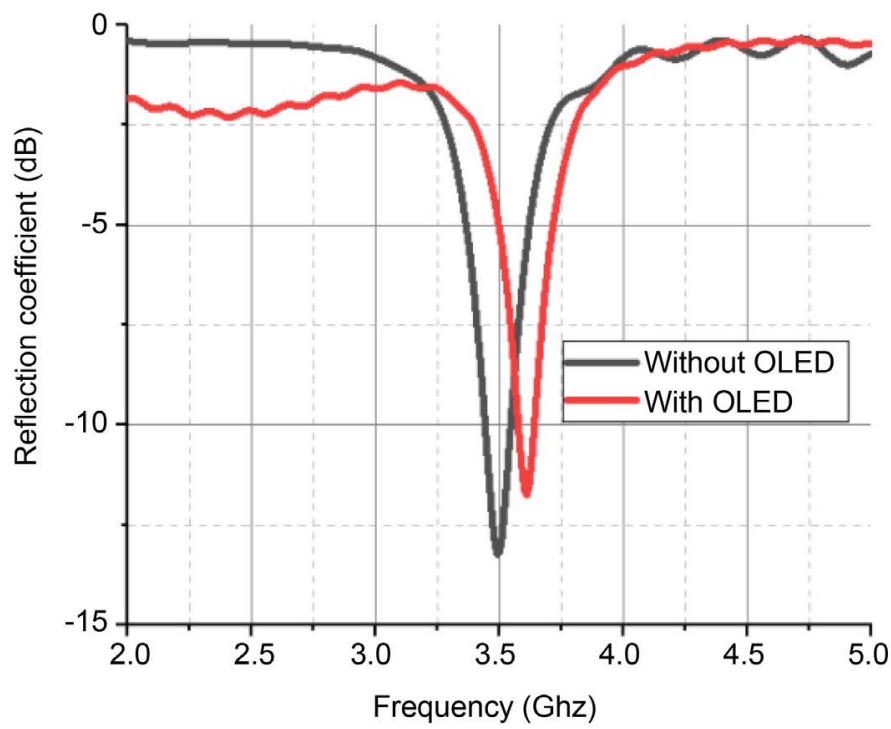

Figure 8. Evaluation of the reflection coefficient (S11) with and without OLED. 


\subsection{Evaluation of the Radiation Pattern before and after OLED Structure Integration}

Figure 9 shows the results of the radiation pattern with and without the integration of the OLED at the resonant frequency of the antenna $\left(f_{R}=3.5 \mathrm{GHz}\right)$.

The radiation patterns in polar coordinates in the $\mathrm{E}$ plane, figure (a), and in the $\mathrm{H}$ plane, figure (b), show the variations depending on the influence of the OLED. The two planes $\mathrm{E}$ and $\mathrm{H}$ show that the radiation patterns are in the same direction and oriented along the $\mathrm{Oz}$ axis. Therefore, this direction represents the same direction of light emission in OLEDs.

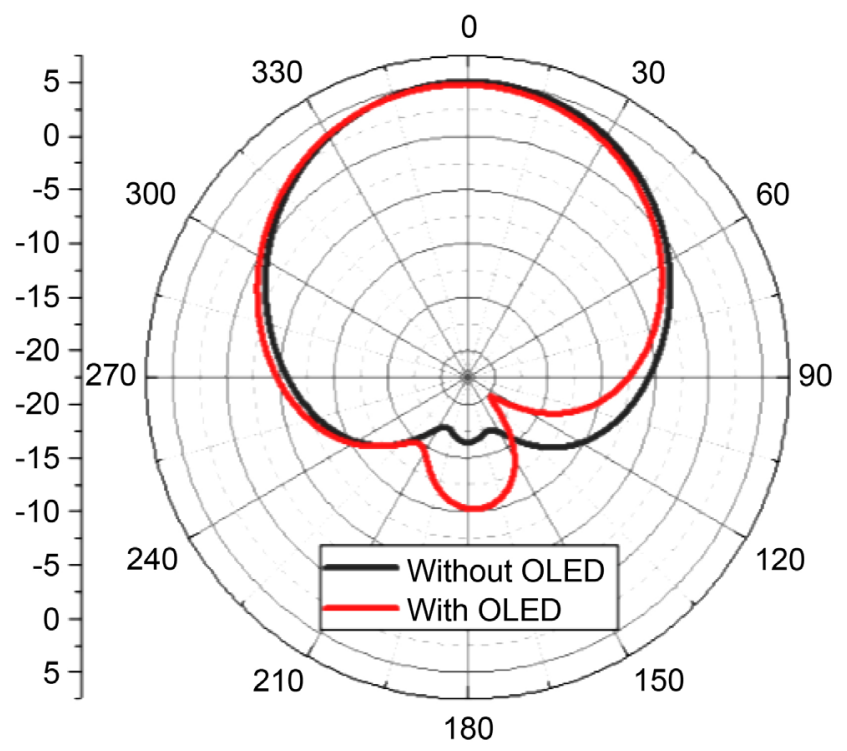

(a)

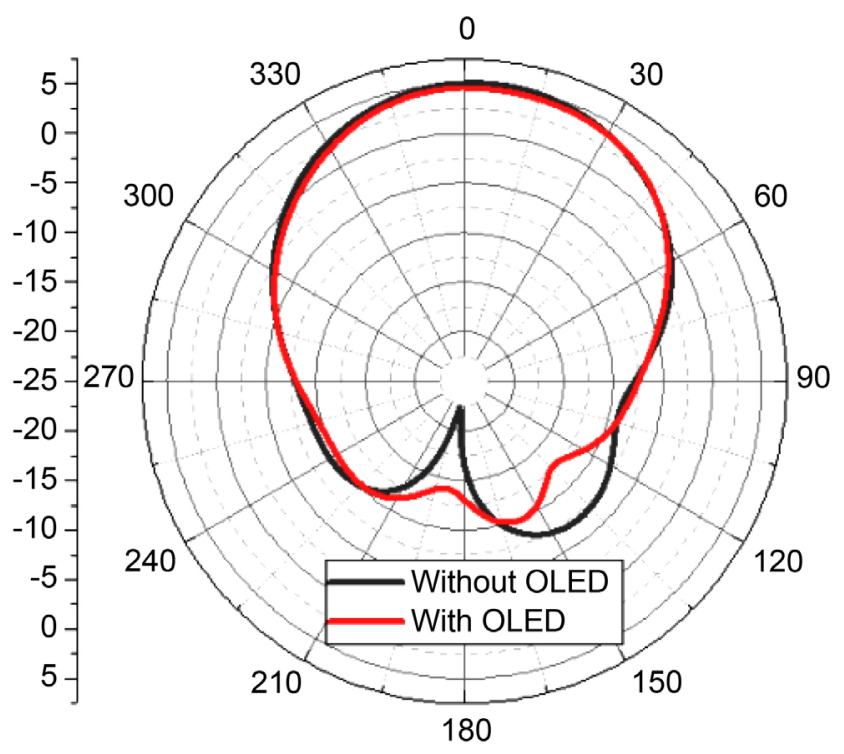

(b)

Figure 9. Radiation pattern for (a) E-Plane and (b) H-Plane, with and without OLED substrate at $3.5 \mathrm{GHz}$. 
The two planes $\mathrm{E}$ and $\mathrm{H}$ represent almost the same directions of the orientation of the radiation of the antenna without and with the light source the OLED with a gain of $4.61 \mathrm{~dB}$. This gain value is lower than that before the integration of the antenna in the OLED. This reduction justifies the influence of the OLED layers on the behavior of the antenna since they have different materials and electrical properties. Bending of the Flexible Antenna Integration in OLED Structure.

\subsection{Bending of the Flexible Antenna integrated in the OLED Structure}

In general, the bends of an antenna can be considered by applying different approaches: 1) fix the dimensions of the size of the antenna without changing them and apply the bending; 2) apply the bending conditions and modify the size of the patch to each of the bending situations and re-tune the offset of the new frequency to the resonant frequency of the flat antenna; and 3) incorporating an adaptive concept, for example. Reconfiguration mechanism, which allows the patch antenna to be re-tuned for each of the bending conditions [26]. Textile antennas are mainly designed to be bent or even applied to specific surfaces during operation, therefore the study of different bending conditions is necessary. Thus, in this part, we will study the impact of bending on the performance of our flexible antenna integrated with OLED by applying approach (a) in this study. The proposed antenna is subjected to a single concave bending position used in this study as shown in Figure 10.

Figure 11 shows the effect of concave bending on the reflection coefficient of the proposed flexible antenna along the $\mathrm{X}$ axis taking into account the different bending angles $30^{\circ}, 45^{\circ}$ and $60^{\circ}$.

The simulation results of the proposed flexible antenna for the concave bends of the three angle values $30^{\circ}, 45^{\circ}$ and $60^{\circ}$ result in small differences in resonant frequency, i.e., the higher the bending angle, the higher the detuning will be. We can see that as the angle of inflection increases (towards a concave structure), the resonant frequency slips to higher frequencies and the reflection coefficient has a better adaptation. However, the bandwidth width remained roughly the same in all bending simulation situations. Despite these small changes observed, the performance of the reflection coefficient $S_{11}$ for the various bending angle values of the proposed antenna remains optimal.

To explain the relation between the angle of curvature and the resonant frequency for the textile antennas observed in Figure 11 we use the Equation (6) cited in [27].

$$
\theta=\frac{W_{\text {antenna }}}{R}
$$

where $\theta$ is the bending angle in radians, $W_{\text {antenna }}$ is the antenna width, and $R$ presents the radius of an imaginary cylinder to which the antenna is bent.

From the Relation (6), we notice that the angle of curvature depends on the 
width of the antenna i.e., more than the angle of curvature increases the width of the antenna also increases which leads to a reduction of the resonant frequency using Equation (1).

In our case the resonance frequency has increased instead of reduced. This behavior can be explained by the integration of our antenna with the OLED, in other words the influence of the materials constituting this OLED on the performance of the antenna and more precisely on the increase in the resonant frequency of our antenna.

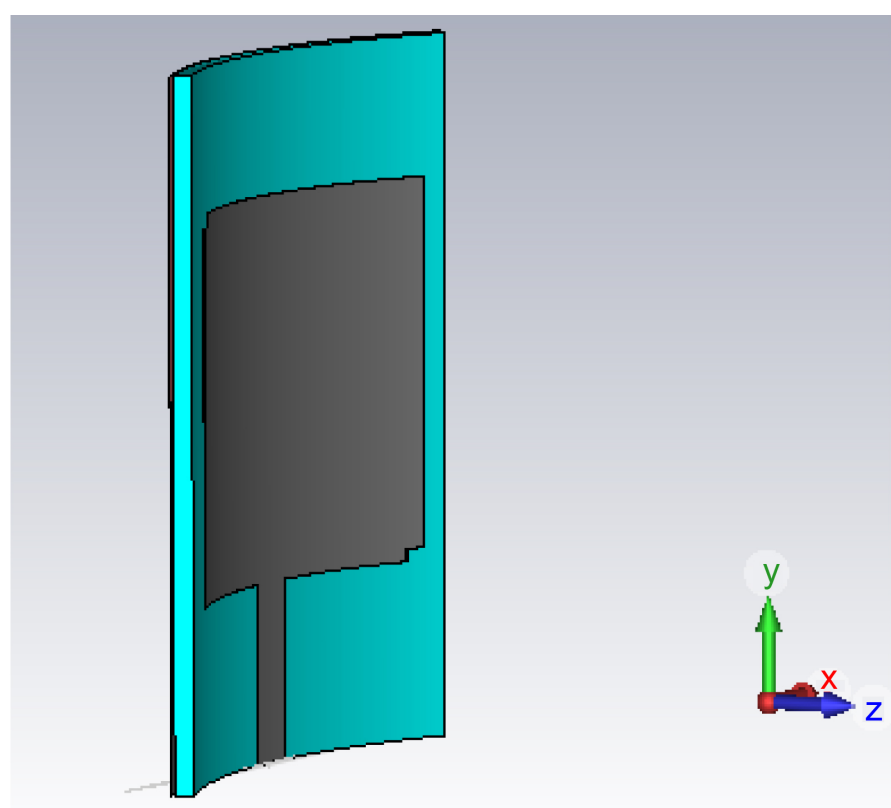

(a)

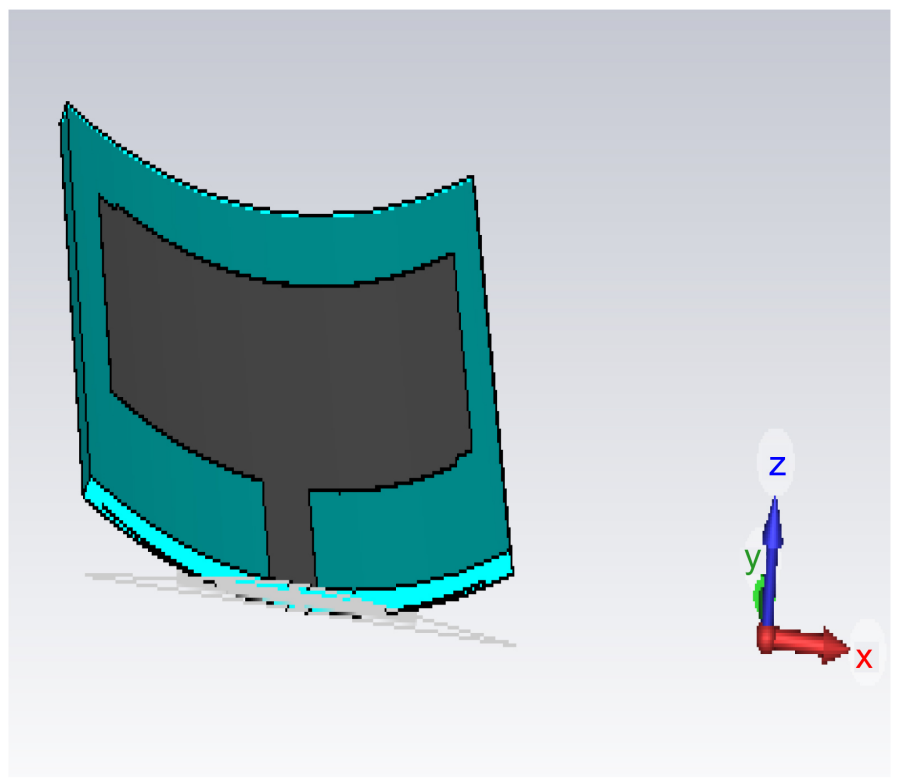

(b)

Figure 10. Concave bending of flexible antenna (a); Perspective (b); Bottom. 


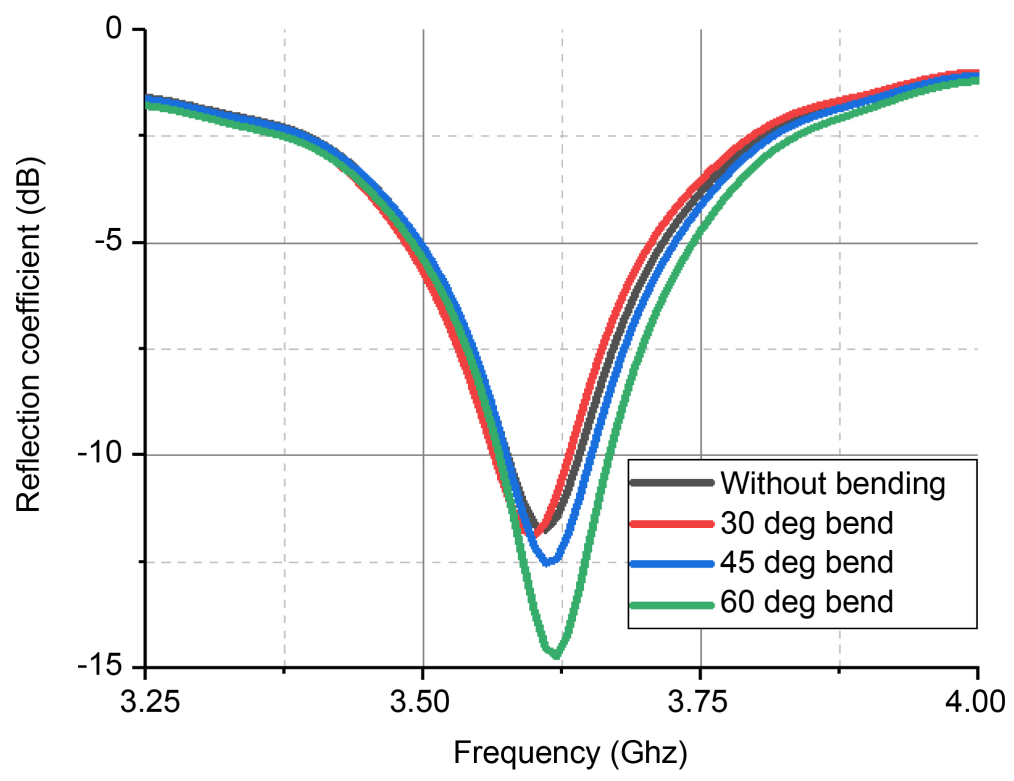

Figure 11. Reflection coefficient of the flexible antenna under the effect of concave bending.

\section{Conclusions}

In this study, carried out using Ansys High Frequency Structure Simulator (Ansys-HFSS) software, we presented a new antenna made from a transparent conductive fabric and we integrated it on OLED lighting sources. These antennas have been adapted to a resonance frequency of $3.5 \mathrm{GHz}$ for use in WIMAX wireless communication systems. The antenna, simple and small in size, is made of VeilShield ${ }^{\mathrm{TM}}$ fabric which allows it to be flexible. We simulated and presented the main parameters (reflection coefficient, gain and radiation pattern) under different conditions with and without the OLED substrate and with and without curvature. The flexible antenna has a high gain of $5.38 \mathrm{~dB}$ at the frequency of $3.5 \mathrm{GHz}$. The results obtained after integration of the antenna show that the OLED lighting source has little effect on the device and that it is possible to produce and integrate this type of flexible antenna on OLEDs. Furthermore, we have also demonstrated that the usual patch antenna design techniques also allow the development of special antennas using unconventional materials.

In conclusion, we were able to demonstrate that it was possible to develop and apply flexible and transparent antennas made from conductive fabrics by integrating them into OLED lighting sources for wireless telecommunications of the WIMAX type.

The research perspectives for this future work in relation to this study involve both the technical implementation of the antenna on a flexible OLED under different experimental conditions including the radiometric, mechanical and photometric characterizations.

\section{Acknowledgements}

This work is supported by a grant from the PHC Maghreb (Hubert Curien Pro- 
gram) MELINA (Mastering Efficient Lighting in North Africa). Funding is provided by Campus France (Grant number 43981ZG awarded to L.C.) and the French Ministries of Europe and Foreign Affairs, and in Morocco, by the Ministry of Higher Education and Scientific Research, in partnership with the National Center for Scientific and Technical Research (CNRST).

\section{Conflicts of Interest}

The authors declare no conflicts of interest regarding the publication of this paper.

\section{References}

[1] Bagher, A.M. (2014) OLED Display Technology. American Journal of Optics and Photonics, 2, 32-36. https://doi.org/10.11648/j.ajop.20140203.13

[2] D’Andrade, B. and Forest, S. (2006) Novel Smart Windows Based on Transparent Phosphorescent OLEDs. Universal Display Corporation, Ewing.

https://doi.org/10.2172/912695

[3] Canale, L. (2019) Imaginons l'éclairage du Futur. Dossier Mieux Voir Et Bien-Être, Magazine LUX.

[4] Zeng, F. (2020) Key Matrix Design Based on OLED Display Technology. 2020 IEEE International Conference on Power, Intelligent Computing and Systems (ICPICS), Shenyang, 28-30 July 2020, 474-479.

https://doi.org/10.1109/ICPICS50287.2020.9202173

[5] Hong, W., Ko, S., Kim, Y.G. and Lim, S. (2017) Invisible Antennas Using Mesoscale Conductive Polymer Wires Embedded within OLED Displays. 2017 11 th European Conference on Antennas and Propagation (EUCAP), Paris, 19-24 March 2017, 28092811. https://doi.org/10.23919/EuCAP.2017.7928446

[6] Hong, W., Lim, S., Ko, S. and Kim, Y.G. (2017) Optically Invisible Antenna Integrated within an OLED Touch Display Panel for IoT Applications. IEEE Transactions on Antennas and Propagation, 65, 3750-3755.

https://doi.org/10.1109/TAP.2017.2705127

[7] El Halaoui, M., Canale, L., Asselman, A. and Zissis, G. (2020) An Optically Transparent Antenna Integrated in OLED Light Source for 5G Applications. 2020 IEEE International Conference on Environment and Electrical Engineering and 2020 IEEE Industrial and Commercial Power Systems Europe (EEEIC/ I\&CPS Europe), Madrid, 9-12 June 2020, 1-5. https://doi.org/10.1109/EEEIC/ICPSEurope49358.2020.9160811

[8] Park, J., et al. (2020) OLED Display-Integrated Optically Invisible Phased Arrays for Millimeter-Wave 5G Cellular Devices. 2020 IEEE/MTT-S International Microwave Symposium (IMS), Los Angeles, 4-6 August 2020, 699-702.

https://doi.org/10.1109/IMS30576.2020.9223830

[9] Sekkal, S., Canale, L. and Asselman, A. (2020) Flexible Textile Antenna Design with Transparent Conductive Fabric Integrated in OLED for WiMAX Wireless Communication Systems. 2020 IEEE International Conference on Environment and Electrical Engineering and 2020 IEEE Industrial and Commercial Power Systems Europe (EEEIC/ I\&CPS Europe), Madrid, 9-12 June 2020, 1-4. https://doi.org/10.1109/EEEIC/ICPSEurope49358.2020.9160757

[10] Zhang, S., et al. (2012) Embroidered Wearable Antennas Using Conductive Threads 
with Different Stitch Spacings. 2012 Loughborough Antennas \& Propagation Conference ( $L A P C$ ), Loughborough, 12-13 November 2012, 1-4. https://doi.org/10.1109/LAPC.2012.6403059

[11] Cicchetti, R., Miozzi, E. and Testa, O. (2017) Wideband and UWB Antennas for Wireless Applications: A Comprehensive Review. International Journal of Antennas and Propagation, 2017, Article ID: 2390808. https://doi.org/10.1155/2017/2390808

[12] Ha, S.-J. and Jung, C.W. (2011) Reconfigurable Beam Steering Using a Microstrip Patch Antenna with a U-Slot for Wearable Fabric Applications. IEEE Antennas and Wireless Propagation Letters, 10, 1228-1231. https://doi.org/10.1109/LAWP.2011.2174022

[13] Yang, L., Rida, A., Vyas, R. and Tentzeris, M.M. (2007) RFID Tag and RF Structures on a Paper Substrate Using Inkjet-Printing Technology. Transactions on Microwave Theory and Techniques, 55, 2894-2901. https://doi.org/10.1109/TMTT.2007.909886

[14] Sankaralingam, S., Dhar, S. and Gupta, B. (2012) Preliminary Studies on Performance of a $2.45 \mathrm{GHz}$ Wearable Antenna in the Vicinity of Human Body. 2012 International Conference on Communications, Devices and Intelligent Systems (CODIS), Kolkata, 28-29 December 2012, 250-253. https://doi.org/10.1109/CODIS.2012.6422185

[15] El Gharbi, M., Fernández-García, R., Ahyoud, S. and Gil, I. (2020) A Review of Flexible Wearable Antenna Sensors: Design, Fabrication Methods, and Applications. $\mathrm{Ma}$ terials (Basel), 13, 3781. https://doi.org/10.3390/ma13173781

[16] Hertleer, C., Rogier, H., Vallozzi, L. and Van Langenhove, L. (2009) A Textile Antenna for Off-Body Communication Integrated into Protective Clothing for Firefighters. IEEE Transactions on Antennas and Propagation, 57, 919-925. https://doi.org/10.1109/TAP.2009.2014574

[17] Balanis, C.A. (2005) Chapter 3. Antenna Theory: Analysis and Design. 3rd Edition, John Wiley Sons, Inc., Hoboken.

[18] Less EMF. Stretch Conductive Fabric. VielshieldTM Conductive Fabric. https://www.lessemf.com/fabric3.html\#11270

[19] Hernandez, E., Nowack, B. and Mitrano, D.M. (2017) Polyester Textiles as a Source of Microplastics from Households: A Mechanistic Study to Understand Microfiber Release during Washing. Environmental Science \& Technology, 51, 7036-7046. https://doi.org/10.1021/acs.est.7b01750

[20] Martin Rey, S. and Melodia, F.R. (2010) Mechanical Strength Parameters on Polyester Fabrics. Treatment Applied in Support of Large Canvas Painting: "The Expulsion of the Merchants from the Temple" by Artist Saverio Lillo. (1734-1796). Arché, No. 4-5, 139-146.

[21] Lee, J., Lee, P., Lee, H., Lee, D., Lee, S.S. and Ko, S.H. (2012) Very Long Ag Nanowire Synthesis and Its Application in a Highly Transparent, Conductive and Flexible Metal Electrode Touch Panel. Nanoscale, 4, 6408-6414. https://doi.org/10.1039/c2nr31254a

[22] Kim, Y.G. (2009) Large-Scale Pattern Growth of Graphene Films for Stretchable Transparent Electrodes. Nature, 457, 706-710. https://doi.org/10.1038/nature07719

[23] Sekitani, T., et al. (2009) Stretchable Active-Matrix Organic Light-Emitting Diode Display Using Printable Elastic Conductors. Nature Materials, 8, 494-499. https://doi.org/10.1038/nmat2459

[24] Garnett, E.C., et al. (2012) Self-Limited Plasmonic Welding of Silver Nanowire Junctions. Nature Materials, 11, 241-249. https://doi.org/10.1038/nmat3238

[25] Alchaddoud, A., Canale, L., Ibrahem, G. and Zissis, G. (2018) Photometric and Electrical Characterizations of Large-Area OLEDs Aged under Thermal and Electrical 
Stresses. IEEE Transactions on Industry Applications, 55, 991-995.

https://doi.org/10.1109/TIA.2018.2864939

[26] Song, L. and Rahmat-Samii, Y. (2018) A Systematic Investigation of Rectangular Patch Antenna Bending Effects for Wearable Applications. IEEE Transactions on Antennas and Propagation, 66, 2219-2228.

https://doi.org/10.1109/TAP.2018.2809469

[27] Ferreira, D., Pires, P., Rodrigues, R. and Caldeirinha, R.F.S. (2017) Wearable Textile Antennas: Examining the Effect of Bending on Their Performance. IEEE Antennas and Propagation Magazine, 59, 54-59. https://doi.org/10.1109/MAP.2017.2686093 